Wioletta Szywacz, Sylwia Mielcarska, Małgorzata Poręba, Agata Macionga, Kamila Stopińska, Nikola Szweda-Gandor, Władysław Grzeszczak

\title{
MC4R polymorphism in rs17782313 influences on insulin resistance
}

\section{Corresponding author:}

Wioletta Szywacz, SPSK No 1, 3-go Maja 13/15 Str., 41-800 Zabrze e-mail: wiolettaszywacz@gmail.com
Medical Research Journal 2021;

Volume 6, Number 2, 94-98

DOI: 10.5603/MRJ.a2021.0023

Copyright (C) 2021 Via Medica

ISSN 2451-2591

e-ISSN 2451-4101

\begin{abstract}
Introduction: There are many factors responsible for the development of metabolic syndrome - mainly associated with lifestyle, but also genetic ones. MC4R (melanocortin 4 receptor) genes variants have been associated with the risk of developing obesity, type 2 diabetes mellitus and coronary artery disease. Aim of the study: To investigate the association between MC4R rs17782313 polymorphism and concentrations of glucose, insulin, HOMA-IR and QUICKI values in the whole study group.

Materials and methods: Study group consisted of 294 patients (136 men and 158 women). Collected venous blood samples were stored at $-70^{\circ} \mathrm{C}$ until the study group was completed. In the laboratory of Clinical Hospital 1 in Zabrze, the DNA materials were isolated, proper concentration of the DNA (15 ng/ $\mu \mathrm{L})$ were prepared and quality and quantity were checked by spectrophotometry. Allelic discrimination was performed with the use of fluorescent-labelled TaqMan Pre-designed SNP Genotyping Assay probes.

Results: No statistically significant differences in concentrations of cholesterol, HDL, LDL, TG between genotypes in women and men were observed. In the whole group of patients, glucose and insulin levels did not differ significantly between TT, CT and CC carriers. Significant differences in values of HOMA-IR and QUICKI between TT, CT and CC carriers as well as between TT carriers and CT + CC carriers were found. CC+TT carriers have a significantly lower value of HOMA-IR and higher QUICKI value than TT carriers. Conclusions: MC4R polymorphism in rs17782313 may be associated with insulin resistance. Further studies are necessary to completely assess the association between investigated polymorphism, insulin resistance and risk of diabetes mellitus development.
\end{abstract}

Key words: melanocortin 4 receptor, insulin resistance, diabetes mellitus, single nucleotide polymorphism, QUICKI, HOMA-IR

Med Res J 2021; 6 (2): 94-98

\section{Introduction}

Abdominal obesity, insulin resistance, raised blood pressure, reduced serum concentration of high-density lipoprotein and elevated serum concentration of triglycerides are gathered as metabolic syndrome, which is closely linked to a high risk of developing cardiovascular diseases and diabetes mellitus. The development of metabolic syndrome is associated both with environmental and genetic factors which influence obesity, glucose and insulin metabolism ${ }^{1}$. Prevalence depends on gender, age socioeconomic status, and ethnic group. Many studies reported that one of the most important factors necessary for metabolic syndrome development is abdominal obesity [1]. Due to the rising prevalence of obesity, the prevalence of metabolic syndrome also increases dramatically; it is estimated that $34 \%$ of women and $50 \%$ of men have MS in Poland [2]. The main cause of insulin resistance, which leads to hyperglycaemia, is an increased level of plasma fatty acids released from adipose tissue [1]. Thus, new markers involved in metabolic syndrome development are still being sought. MC4R (melanocortin 4 receptor) is the G-protein coupled receptor that binds Pro-opiomelanocortin (POMC)-derived melanocortin peptides and transmits satiety signal in the paraventricular nucleus [3]. Its gene is located on the long arm of chromosome 18. MC4R is considered an important regulator of food intake and metabolism which dysfunction may be associated with the develop- 
ment of obesity, insulin resistance, type 2 diabetes and coronary artery disease [4]. Many SNP near the MC4R gene are reported to be associated with an increased risk of obesity $[5,6]$.

Insulin resistance $(\mathrm{IR})$ is described as a disorder in the regulation of glucose homeostasis which is manifested by decreased insulin ability to reduce serum glucose level despite its normal or increased level in serum [7]. This pathological condition is caused by decreased sensitivity of muscles, adipose tissue, liver, and other body tissues to insulin [7]. The insulin sensitivity resistance is often assessed using Homeostasis Model Assessment — Insulin Resistance (HOMA-IR). To assess insulin sensitivity which is the inverse of insulin resistance the quantitative insulin sensitivity check index (QUICKI) is used [7].

The study aimed to investigate the association between different variants of the MC4R gene in rs17782313 and serum concentrations of glucose, insulin, HDL, LDL, TG, HOMA-IR and QUICKI values in a group of Polish patients (the industrial region of Silesia, Poland).

\section{Materials and methods}

\section{Study sample}

294 study samples of whole blood were collected. Inclusion criteria contained: age $>18$ years, no tumour, no dialysis at the time of sampling and informed consent. Patients were included in the study by a random selection.

\section{Evaluation of DNA levels}

Whole blood samples were collected for the examination, then isolated by column method. Afterwards, they were stored until the study group was collected. The concentration of genetic material $15 \mathrm{ng} / \mu \mathrm{L}$ was obtained by mixing DNA with water according to the dilution protocol and checked with a denoviX spectrophotometer.

\section{Preparation of samples}

Preparation for PCR included: preparation of the mixture by vortexing on a short spin probe, mixing with water, buffer and mix (reaction mix to Roche device), re-vortexing, centrifugation. The solution was mixed with the DNA and transported to the PCR plate. PCR reaction was performed by Roche Lightcycler 96. Alleles were marked as A in VIC and G in FAM (Tab. 1).

\section{Statistical analysis}

The Shapiro-Wilk test was used to assess the data distribution. Variables data are presented as mean $\pm S D$. The significance between distributions of genotypes and alleles, gender, the occurrence of hypertension, coronary artery disease (MIC), diabetes mellitus, cigarettes smoking was tested using Pearson's $\chi 2$ test. To compare valuable data between the three groups (genotypes TT, CT and CC), the Kruskal-Wallis test with multiple comparisons was performed. To compare valuable data between two groups (groups TT, CT+CC) Mann-Whitney $U$ test was performed. Concentrations of cholesterol, HDL, LDL, TG were analysed separately for women and men. Levels of glucose, insulin, values of HOMA-IR and QUICKI were analysed for the whole study group.

\section{Results}

No statistically significant differences were found in concentrations of cholesterol, HDL, LDL, TG between genotypes in groups of women and men (Tab. 2, 3).

In the whole group of patients, glucose and insulin levels did not differ significantly between TT, CT and CC carriers (Tab. 4). Significant differences in values of $\mathrm{HO}-$ MA-IR and QUICKI between TT, CT and CC carriers as well as between $\mathrm{TT}$ carriers and CT+CC carriers were found. $\mathrm{CC}+\mathrm{CT}$ carriers have a significantly lower value of $\mathrm{HO}-$ MA-IR and higher QUICKI value than TT carriers (Fig. 1, 2).

\section{Discussion}

Many studies reported a significant association between MC4R SNP in rs17782313 and parameters of metabolic syndrome, however, the results were often inconclusive. Research conducted by Brodowski et al. revealed that the probability of MS development in a group of postmenopausal women is higher in individuals with the CT or CC genotype in rs17782313. Women with CT or CC genotype had higher levels of TG, total cholesterol, LDL-C and Apolipoprotein B than those with TT genotype ${ }^{8}$. On the contrary, the study carried out by Szkup et al. reported that MC4R SNP in rs17782313 is not linked to a higher risk of metabolic syndrome development in a group of 45-60-year-old women [9]. H. Yarizadeh et al. showed that in the population of overweighted or obese Iranian women the presence of allele $\mathrm{C}$ in rs 17782313 is associated with higher HOMA-IR value and elevated insulin level [10]. Contrary to these results, the presented study found significantly higher HOMA-IR value in patients with TT genotype compared to TC and CC individuals. The differences in HOMA-IR and QUICKI value between genotypes were insignificant in the study conducted by Brodowski [8] and not assessed in research conducted by Szkup [9]. The authors assume that the presence of allele T is associated with increased HOMA-IR value 
Table 1. Characteristic of study group $(n=294)$

\begin{tabular}{|c|c|c|c|c|c|c|c|}
\hline & \multicolumn{6}{|c|}{ Genotype } & \multirow{3}{*}{$\mathbf{p}$} \\
\hline & \multicolumn{2}{|c|}{ TT } & \multicolumn{2}{|c|}{ CT } & \multicolumn{2}{|c|}{ CC } & \\
\hline & $\mathbf{n}$ & $\%$ & $\mathbf{n}$ & $\%$ & $\mathbf{n}$ & $\%$ & \\
\hline Gender & & & & & & & 0.253 \\
\hline Women & 103 & $63.58 \%$ & 53 & $32.72 \%$ & 2 & $1.23 \%$ & \\
\hline Men & 85 & $61.59 \%$ & 45 & $32.61 \%$ & 6 & $4.35 \%$ & \\
\hline Hypertension & & & & & & & 0.46039 \\
\hline Yes & 96 & $65.75 \%$ & 44 & $30.14 \%$ & 5 & $3.42 \%$ & \\
\hline No & 92 & $59.74 \%$ & 54 & $35.06 \%$ & 3 & $1.95 \%$ & \\
\hline MIC & & & & & & & 0.34531 \\
\hline Yes & 31 & $63.27 \%$ & 15 & $30.61 \%$ & 3 & $6.12 \%$ & \\
\hline No & 157 & $62.55 \%$ & 83 & $33.07 \%$ & 5 & $199.20 \%$ & \\
\hline Diabetes mellitus & & & & & & & 0.36361 \\
\hline Yes & 21 & $70.00 \%$ & 9 & $30.00 \%$ & 0 & $0.00 \%$ & \\
\hline No & 167 & $61.85 \%$ & 89 & $32.96 \%$ & 8 & $2.96 \%$ & \\
\hline Nicotinism & & & & & & & 0.66682 \\
\hline Yes & 41 & $57.75 \%$ & 26 & $36.62 \%$ & 2 & $2.82 \%$ & \\
\hline \multirow[t]{2}{*}{ No } & 147 & $64.19 \%$ & 72 & $31.44 \%$ & 6 & $2.62 \%$ & \\
\hline & mean & SD & mean & SD & mean & SD & $p$ \\
\hline Age & 53,66 & 16.84 & 51.26 & 16.92 & 58.88 & 18.01 & 0.1929 \\
\hline $\mathrm{BMI}$ & 26,80 & 3.70 & 26.49 & 4.03 & 25.18 & 1.77 & 0.2554 \\
\hline
\end{tabular}

$\mathrm{BMI}$ - body mass index, MIC — coronary artery disease

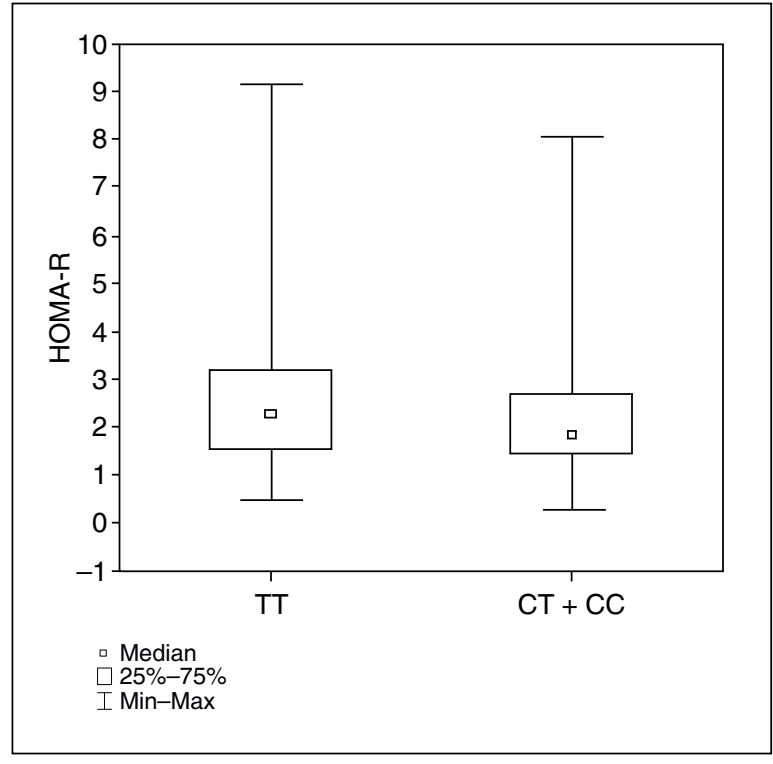

Figure 1. Comparison of HOMA-IR value between TT carriers (wild genotype) and CC or CT carriers ( $p=0,036, n=294$ ) HOMA-IR - homeostasis model assessment-insulin

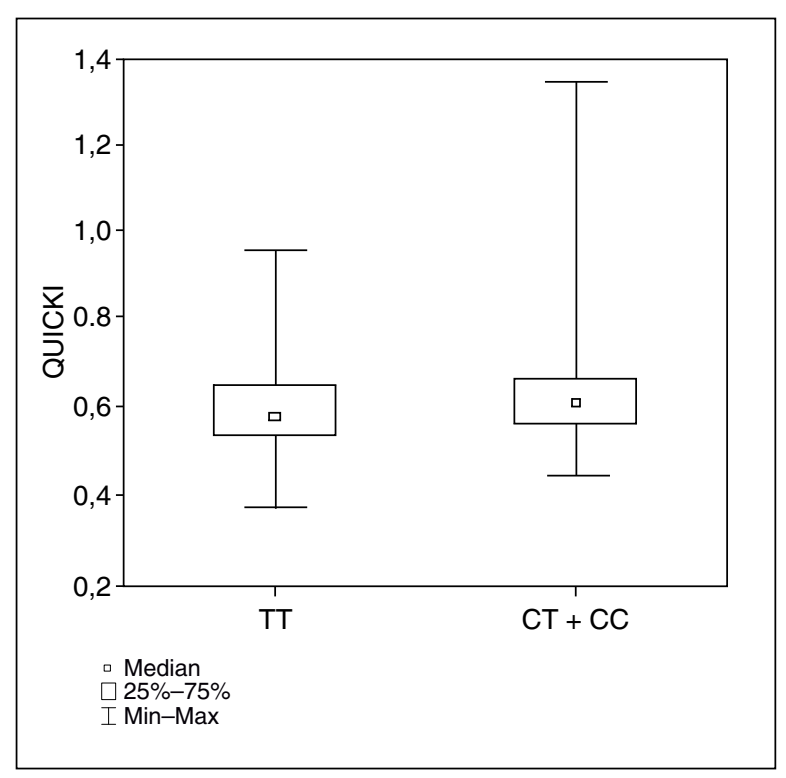

Figure 2. Comparison of QUICKI value between TT carriers (wild genotype) and CC or CT carriers $(p=0,018, n=294$ ) QUICKI - quantitative insulin sensitivity check index 
Table 2. MC4R rs17782313 polymorphism and cholesterol, HDL, LDL, TG levels in women $(n=167)$

\begin{tabular}{|c|c|c|c|c|c|c|c|}
\hline & \multicolumn{6}{|c|}{ Women } & \multirow{3}{*}{$p$} \\
\hline & \multicolumn{2}{|c|}{ TT } & \multicolumn{2}{|c|}{ CT } & \multicolumn{2}{|c|}{ CC } & \\
\hline & mean & SD & mean & SD & mean & SD & \\
\hline Cholesterol & 6.05 & 1.16 & 6.46 & 1.21 & 6.58 & 0.57 & 0.1747 \\
\hline HDL & 1.34 & 0.35 & 1.44 & 0.30 & 1.23 & 0.86 & 0.1946 \\
\hline LDL & 4.09 & 1.04 & 4.45 & 1.13 & 4.90 & 0.21 & 0.1179 \\
\hline TG & 1.37 & 0.80 & 1.24 & 0.54 & 1.00 & 0.18 & 0.461 \\
\hline
\end{tabular}

HDL — high-density lipoprotein, LDL — low-density lipoprotein, MC4R - melanocortin 4 receptor, TG — triacylglycerols

Table 3. MC4R rs17782313 polymorphism and cholesterol, HDL, LDL, TG levels in men $(n=142)$

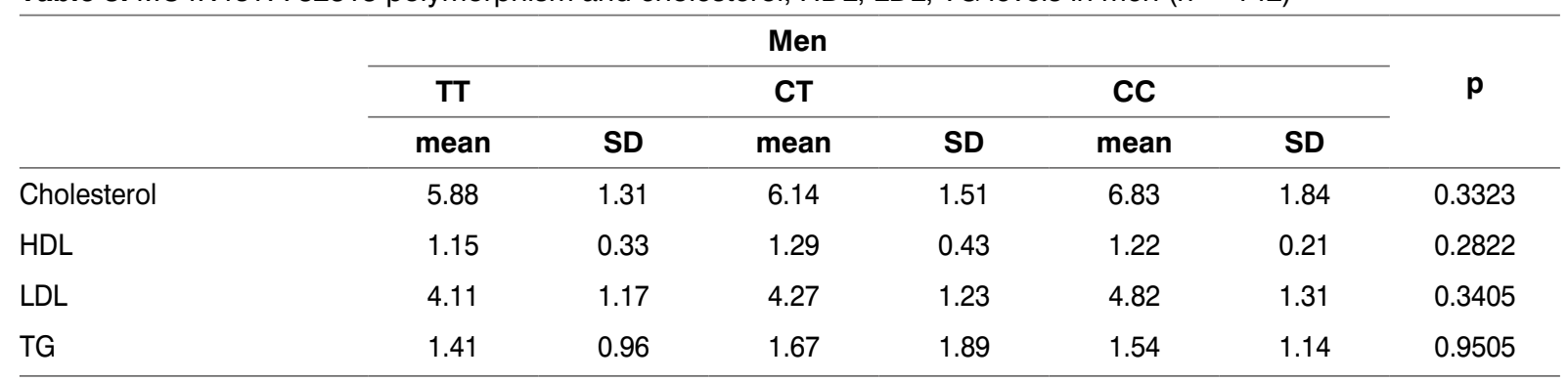

HDL — high-density lipoprotein, LDL — low-density lipoprotein, MC4R - melanocortin 4 receptor, TG — triacylglycerols

Table 4. MC4R rs17782313 polymorphism and concentrations of glucose, insulin, HOMA-IR and QUICKI values in the whole study group $(n=294)$

\begin{tabular}{lccccccc}
\hline & \multicolumn{2}{c}{ TT } & \multicolumn{2}{c}{ CT } & \multicolumn{2}{c}{ CC } & p \\
\cline { 2 - 7 } & mean & SD & mean & SD & mean & SD & \\
\hline Glucose & 84.81 & 24.16 & 80.42 & 16.05 & 73.25 & 10.94 & 0.0891 \\
Insulin & 13.06 & 9.74 & 11.39 & 5.72 & 7.98 & 3.49 & 0.0364 \\
HOMA-IR & 2.51 & 1.39 & 2.31 & 1.43 & 1.42 & 0.62 & 0.0135 \\
QUICKI & 0.60 & 0.10 & 0.62 & 0.12 & 0.69 & 0.09 & 0.0079 \\
\hline
\end{tabular}

HOMA-IR — homeostasis model assessment-insulin, MC4R - melanocortin 4 receptor, QUICKI — quantitative insulin sensitivity check index

and insulin resistance, however, the differences in genotype distributions between diabetes and nondiabetic patients were insignificant. One must bear in mind that other studies tested different models of inheritance, thus contradictory results can depend on the used model. This study used the dominant model of the MC4R rs17782313 inheritance (TT, CT+CC).

In the presented work no significant association between MC4R SNP in rs17782313 and obesity was found. This observation is in line with two similar studies conducted in Poland $[9,11]$ and one carried out in the Czech Republic [12]. On the contrary, other population studies conducted in Pakistan [13] and Greece [14] showed that homozygotes CC have a higher risk of obesity; similarly, in the Romania population heterozygotes CT are more predisposed to obesity [15]. It can be supposed that the presence of allele $C$ is associated with an increased risk of obesity. However, due to differences in research findings, further studies are necessary to thoroughly investigate the influence of SNP MC4R on the prevalence of overweight and obesity. One should also take into account the role of population characteristics which can be responsible for the lack of association between SNP of MC4R and obesity in the Polish population.

Lipid profile disturbances as a component of metabolic syndrome were also analysed in the conducted research. Similar to this study, other studies conducted in Poland [9, 11] and Greece [14] did not find a statistically significant association between MC4R SNP in rs17782313 and the lipid profile of patients. There is only one study reporting a reduced risk of lipid disturbances among individuals with $\mathrm{T}$ allele in a group of Polish postmenopausal women [8]. 
Some studies demonstrated a relationship between MC4R SNP in rs17782313 and glycaemic profile - fasting blood glucose and insulin level. Yarizadeh et al. showed significantly higher insulin level in CT or CC carriers in comparison to TT carriers [10]. However, a similar study conducted by Lazopoulou et al. in the Greek population did not reveal the association between MC4R SNP in rs17782313 and insulin or glucose level [14]. This research has found no association between investigated SNP and glucose or insulin concentration.

The most significant results of this study concern the statistical association between the parameters measuring insulin resistance (HOMA-IR and QUICKI) and the studied SNP. In the studied population of women aged 45-60, no correlation was found between this parameter and metabolic syndrome [9]. Further studies in both genders are necessary to confirm or rule out the association between insulin resistance and MC4R SNP.

\section{Conclusion}

The obtained results showed that SNP in rs17782313 of MC4R gene is not significantly associated with lipid profile disturbances which are characteristic of metabolic syndrome. However, statistically significant differences in HOMA-IR and QUICKI value may suggest an influence of investigated SNP on insulin resistance. TT genotype was present more often in patients with increased insulin resistance while CT and CC genotypes were associated with decreased insulin resistance and higher insulin sensitivity. No statistically significant association between studied SNP and occurrence of hypertension, coronary artery disease and diabetes mellitus was observed. Further studies with a larger number of patients are needed to clarify the role of SNP in rs 17782313 of MC4R gene in the pathogenesis of metabolic syndrome and insulin resistance which is closely related to this disorder.

Acknowledgements: Authors would like to thank Władysław Grzeszczak and the Department of Internal Medicine, Diabetology and Nephrology in Zabrze for the idea and for helping them financially with the research. The authors wish to show their appreciation to Nikola Szweda-Gandor who supported them in every part of the research. They thank for support in laboratory: Wanda Trautsolt and Sylwia Górczyńska-Kosiorz.

\section{Conflict of interest: None.}

\section{References}

1. Wang HH, Lee DKi, Liu M, et al. Novel Insights into the Pathogenesis and Management of the Metabolic Syndrome. Pediatr Gastroenterol Hepatol Nutr. 2020; 23(3): 189-230, doi: 10.5223/pghn.2020.23.3.189, indexed in Pubmed: 32483543.

2. Janszky I, Vatten L, Romundstad P, et al. Metabolic syndrome in Poland - the PONS Study. Ann Agric Environ Med. 2011; 18(2): 270-272, indexed in Pubmed: 22216795

3. Faroogi IS, O'Rahilly S. Mutations in ligands and receptors of the leptin-melanocortin pathway that lead to obesity. Nat Clin Pract Endocrinol Metab. 2008; 4(10): 569-577, doi: 10.1038/ncpendmet0966, indexed in Pubmed: 18779842

4. Lotta LA, Mokrosinski J, Mendes de Oliveira E, et al. Human gain-offunction MC4R variants show signaling bias and protect against obesity. Yearbook of Paediatric Endocrinology. 2020, doi: 10.1530/ey.17.11.3.

5. Qin Li, Tiwari AK, Zai CC, et al. Regulation of melanocortin-4-receptor (MC4R) expression by SNP rs17066842 is dependent on glucose concentration. Eur Neuropsychopharmacol. 2020; 37: 39-48, doi: 10.1016/j.euroneuro.2020.05.008, indexed in Pubmed: 32684494

6. Wei BL, Yin RX, Liu CX, et al. The MC4R SNPs, their haplotypes and gene-environment interactions on the risk of obesity. Mol Med. 2020; 26(1): 77, doi: 10.1186/s10020-020-00202-1, indexed in Pubmed: 32770936.

7. Gierach MA, Junik R. Insulin resistance in metabolic syndrome depending on the occurrence of its components. Endokrynologia Polska. ; 2021.

8. Brodowski J, Szkup M, Jurczak A, et al. Searching for the relationship between the parameters of metabolic syndrome and the rs 17782313 (TC) polymorphism of the MC4R gene in postmenopausal women. Clin Interv Aging. 2017; 12: 549-55.

9. Małgorzata S, Jacek B, Jerzy OA, et al. Searching for Factors Raising the Incidence of Metabolic Syndrome Among 45-60-Year-Old Women. Aging Dis. 2018; 9(5): 831-842, doi: 10.14336/AD.2017.1027, indexed in Pubmed: 30271660

10. Yarizadeh H, Mirzababaei A, Ghodoosi N, et al. The interaction between the dietary inflammatory index and MC4R gene variants on cardiovascular risk factors. Clin Nutr. 2021; 40(2): 488-495, doi: 10.1016/j. clnu.2020.04.044, indexed in Pubmed: 32586686

11. Rotter I, Skonieczna-Żydecka K, Kosik-Bogacka D, et al. Relationships between rs9939609, rs17782313, and rs1801282 polymorphisms and the occurrence of selected metabolic and hormonal disorders in middle-aged and elderly men - a preliminary study. Clin Interv Aging. 2016; 11: 1723-1732, doi: 10.2147/CIA.S120253, indexed in Pubmed: 27920511.

12. Dušátková $L$, Zamrazilová $H$, Sedláčková $B$, et al. Association of obesity susceptibility gene variants with metabolic syndrome and related traits in 1,443 Czech adolescents. Folia Biol (Praha). 2013; 59(3): 123-133, indexed in Pubmed: 23890480.

13. Rana S, Sultana A, Bhatti AA. Association of rs6265 and rs 17782313 with metabolic syndrome in Pakistanis. J Biosci. 2019; 44(4), indexed in Pubmed: 31502573

14. Lazopoulou N, Gkioka E, Ntalla I, et al. The combined effect of MC4R and FTO risk alleles on childhood obesity in Greece. Hormones (Athens). 2015; 14(1): 126-133, doi: 10.14310/horm.2002.1524, indexed in Pubmed: 25402378.

15. Voiculescu VM, Solomon I, Popa A, et al. Gene polymorphisms of TNF-238G/A, TNF-308G/A, IL10-1082G/A, TNFAIP3, and MC4R and comorbidity occurrence in a Romanian population with psoriasis. J Med Life. 2018; 11: 69-74. 